\title{
A Simple Graphical Way of Evaluating Coverage and Directional Non-coverages*
}

\author{
Adelaide Freitas, Department of Mathematics \& CIDMA, \\ University of Aveiro, Portugal (adelaide@ua.pt) \\ Sara Escudeiro, ?? \\ \& CIDMA, University of Aveiro, Portugal (sarae@ua.pt), \\ Vera Afreixo, Department of Mathematics \& CIDMA, \\ University of Aveiro, Portugal (vera@ua.pt) Portugal
}

\begin{abstract}
Evaluation of the coverage probability and, more recently, of the intervalar location of confidence intervals, is a useful procedure if exact and asymptotic methods for constructing confidence intervals are used for some populacional parameter. In this paper, a simple graphical procedure is presented to execute this kind of evaluation in confidence methods for linear combinations of $k$ independent binomial proportions. Our proposal is based on the representation of the mesial and distal non-coverage probabilities on a plane. We carry out a simulation study to show how this graphical representation can be interpreted and used as a basis for the evaluation of intervalar location of confidence interval methods.
\end{abstract}

Key Words and Phrases: principal component analysis, clustering, $k$-means.

\section{Introduction}

Inference involving more than one population parameter is very common in Statistics. For instance, the effect of the interaction between the presence and absence of two treatments $A$ and $B$ can be established in terms of a linear combination of four independent binomial proportions, $p_{1}-p_{2}-p_{3}+p_{4}$, where each $p_{i}, i=1,2,3$, 4 , denotes the unknown population

*Accepted authors manuscript (AAM) published by Springer in [ICCSA 2017, Part II, LNCS 10405, pp. 600-610, 2017]. [DOI: 10.1007/978-3-319-62395-5_41]. The final publication is available at Springer via http://dx.doi.org/10.1007/978-3-319-62395-5_41 
proportion in one of four possible groups. In order to analyze the existence of interaction between treatments $A$ and $B$, the following statistical test could be carried out:

$$
H_{0}: p_{1}-p_{2}-p_{3}+p_{4}=0 \quad \text { vs } \quad H_{1}: p_{1}-p_{2}-p_{3}+p_{4} \neq 0 .
$$

Moreover, due to the dual relationship between statistical tests and confidence intervals (CIs), this testing problem can also be addressed in terms of the CI for $p_{1}-p_{2}-p_{3}+p_{4}$.

In this paper, we deal with the evaluation of the performance of asymptotic methods used to construct two-sided CIs involving two or more population parameters. In particular, we focus our study on proportions. Asymptotic methods are generally preferred because they are computationally simpler and faster than exact ones. Several approximate methods have been proposed in the literature for constructing confidence intervals (CIs) for the difference of two independent binomial proportions ([1, 2, 3, 4, 5, 6]). However, few authors have been discussing approximate methods for obtaining CIs for any linear combination of two $([7,8])$ and more than two $([9,10,11,12,13])$ independent binomial populations. Within the context of investigating the properties of each of the different approaches to construct CIs, the performance of each method is commonly evaluated through simulation studies. Such evaluations are usually based on the exact coverage probabilities of each method. More recently ([13, 15, 16]), the expected interval location of the CIs, which is based on the mesial and distal non-coverage probabilities, has also been considered as an important performance measure. In the present work we discuss a graphical representation of the two directional non-coverage probabilities, aimed at facilitating the characterization of interval location.

The paper is organized as follows. In Section 2, a brief overview of four variants of the classic Wald CI is provided. In Section 3 , evaluative indexes related to the directional non-coverages are highlighted. In Section 4 we propose a graphical technique to evaluate the CIs location. Section 5 shows some examples of the application of this graphical technique. Finally, we summarize our findings in Section 6 .

\section{Classic and Adjusted Wald CIs for Linear Combinations of Proportions}

Due to the dual relationship between statistical tests and CIs, the most common approach to obtain large-sample interval estimates for a combination $L=\sum_{i=1}^{k} \beta_{i} p_{i}$ of $k \geq 1$ binomial proportions $p_{1}, p_{2}, \ldots, p_{k}$ from independent binomial populations $X_{1}, X_{2}, \ldots, X_{k}$ with $n_{1}, n_{2}, \ldots, n_{k}$ trials, respectively, and weights given by $k$ fixed constants $\beta_{1}, \beta_{2}, \ldots, \beta_{k} \neq 0$, consists in inverting the standard two-sided Wald test $H_{0}: L=\lambda_{0}$, where $\lambda_{0}$ is any real constant admissible for $\sum_{i=1}^{k} \beta_{i} p_{i}$, meaning that $\lambda_{0}$ should belong to the support scale $\left[\sum_{\beta_{i}<0} \beta_{i} ; \sum_{\beta_{i}>0} \beta_{i}\right]$. The general formula of the classic Wald CI is

$$
\hat{L} \mp z_{\alpha / 2} \sqrt{\hat{v}(\hat{L})}
$$


where $z_{\alpha / 2}$ is the $\alpha / 2$ upper quantile of the standard normal distribution and $\hat{L}$ and $\hat{v}(\hat{L})$ represent an estimate of $L$ and the variance of estimator $\hat{L}$, respectively. When variance is estimated using the maximum likelihood estimate (MLE) of each $p_{i}, i=1,2, \ldots, k$, the classic version of the Wald CI, known for its poor coverage properties (e.g. [14, 4, 11]), is obtained. When variance is estimated using a shrinkage estimator for each $p_{i}$ given by

$$
\frac{X_{i}+h_{i}}{n_{i}+2 h_{i}}
$$

for some $h_{i}>0, i=1, \ldots, k$, that is, by adding $h_{i}$ successes and $h_{i}$ failures to the original data, a modified version of the Wald CI, the so-called adjusted Wald CI is obtained. Depending on the particular $h_{i}$ chosen, different variants of the adjusted Wald CI can be established. A list of $h_{i}$ values herein considered and the names given to their corresponding variants is presented below:

- $h_{i}=0 \quad$ (variant-0, classic version)

- $h_{i}=\frac{2}{k} \quad$ (variant-1, [10])

- $h_{i}=\frac{z_{\alpha / 2}^{2}}{2 k} \quad($ variant-2)

- $h_{i}=\frac{z_{\alpha / 2}^{2}}{2}\left(\mathbf{1}_{\mathcal{A}_{i}}\left(x_{i}\right)+\frac{1}{k}\right) \quad($ variant-3, [1] $)$

- $h_{i}=\frac{z_{\alpha / 2}^{2}}{2}\left(\mathbf{1}_{\mathcal{A}_{i}}\left(x_{i}\right)+\frac{\beta_{i}^{2} / n_{i}}{\sum_{i=1}^{k} \frac{\beta_{i}^{2}}{n_{i}}}\right) \quad($ variant-4, [13])

where $\mathbf{1}_{\mathcal{A}_{i}}(\cdot)$ is the indicator function of

$$
\mathcal{A}_{i}=\left\{x_{i} \in\left\{0, n_{i}\right\}:\left(n_{i}-2 x_{i}\right)\left(\hat{L}-\lambda_{0}\right) \beta_{i}<0\right\} .
$$

It is obvious that variant-2 is equal to variant-3 when $0<x_{i}<n_{i}$ for all $i$, variant- 1 is approximately equal to variant-2 when $\alpha=5 \%$, and variant-3 is equal to variant- 4 when $\frac{\beta_{i}^{2}}{n_{i}}$ is a constant for all $i$. The adjusted Wald CIs have better performance than the classic Wald CI.

\section{Coverage and Directional Non-coverages}

In order to assess and compare the performance of methods to construct CIs for any linear combination $L=\sum_{i=1}^{k} \beta_{i} p_{i}$, evaluations of the exact coverage probabilities and locations (characterized by its mesial and distal non-coverage probabilities) can be performed.

Given the weights $\left(\beta_{1}, \beta_{2}, \ldots, \beta_{k}\right)$ and a set of $k$ independent binomials with parameters $\left(n_{1}, p_{1}\right),\left(n_{2}, p_{2}\right), \ldots,\left(n_{k}, p_{k}\right)$, the exact coverage probability $(\mathrm{R})$ can be computed as

$$
\mathrm{R}(L)=\sum_{x_{1}=0}^{n_{1}} \sum_{x_{2}=0}^{n_{2}} \ldots \sum_{x_{k}=0}^{n_{k}} \prod_{i=1}^{k}\left(\begin{array}{c}
n_{i} \\
x_{i}
\end{array}\right) p_{i}^{x_{i}}\left(1-p_{i}\right)^{n_{i}-x_{i}} \mathbf{1}_{[l(\boldsymbol{x}), u(\boldsymbol{x})]}(L)
$$


where $[l(\boldsymbol{x}), u(\boldsymbol{x})]$ is the CI obtained from the observation $\boldsymbol{x}=\left(x_{1}, x_{2}, \ldots, x_{k}\right)$ for the linear combination $L=\sum_{i=1}^{k} \beta_{i} p_{i}$. To examine the interval location, we considered the procedure suggested by [13] for the linear combination. Concretely, for each CI of a linear combination, we analyse the existence of equilibrium between the directions of the mesial non-coverage probability (MNR) and distal non-coverage probability (DNR). These directions indicate whether the CIs are located too distally or too mesially from the midpoint $c$ of the support scale relatively to the true value $L\left(c=\sum_{i=1}^{k} \beta_{i} / 2\right)$. The MNR and DNR are defined as

$$
\operatorname{MNR}(L)=\sum_{x_{1}=0}^{n_{1}} \sum_{x_{2}=0}^{n_{2}} \ldots \sum_{x_{k}=0}^{n_{k}} \prod_{i=1}^{k}\left(\begin{array}{l}
n_{i} \\
x_{i}
\end{array}\right) p_{i}^{x_{i}}\left(1-p_{i}\right)^{n_{i}-x_{i}} \mathbf{1}_{\mathcal{M}}(\boldsymbol{x}),
$$

with $\mathcal{M}=\{\boldsymbol{x}:(L \leq c \wedge u(\boldsymbol{x})<L) \vee(L \geq c \wedge l(\boldsymbol{x})>L)\}$, and

$$
\operatorname{DNR}(L)=\sum_{x_{1}=0}^{n_{1}} \sum_{x_{2}=0}^{n_{2}} \ldots \sum_{x_{k}=0}^{n_{k}} \prod_{i=1}^{k}\left(\begin{array}{c}
n_{i} \\
x_{i}
\end{array}\right) p_{i}^{x_{i}}\left(1-p_{i}\right)^{n_{i}-x_{i}} \mathbf{1}_{\mathcal{D}}(\boldsymbol{x}),
$$

with $\mathcal{D}=\{\boldsymbol{x}:(L<c \wedge l(\boldsymbol{x})>L) \vee(L>c \wedge u(\boldsymbol{x})<L)\}$.

The non-coverage is said to be mesial iff the interval is located too distally to include the true parameter value $L$ (see Fig. 1 (a)) and the non-coverage is said to be distal iff the interval is located too mesially to include the true parameter value $L$ (see Fig. 1 (b)).

According to [15], the interval location of the CIs can be characterized by the ratio

$$
\mathrm{Q}=\frac{\mathrm{MNR}}{1-\mathrm{R}}=\frac{\mathrm{MNR}}{\mathrm{MNR}+\mathrm{DNR}}
$$

This ratio expresses the balance condition between MNR and DNR. Based on a partition of the range of values of $\mathrm{Q}$ (see Fig. 2, on the top), [15] and, more recently, 13] established a classification criterion for the location of CIs for linear combinations of independent binomial proportions. Concretely, values of $\mathrm{Q}$ between 0.375 and 0.625 correspond to satisfactorily located CIs, less than 0.375 to CIs located too mesially to include the true value of $L$, and greater than 0.625 to CIs located too distally to include $L$. Hence, when (see Fig. 2 (a)),

- MNR and DNR are balanced, CIs are satisfactorily located;

- MNR predominates, CIs are too or much too distally located; and,

- DNR predominates, CIs are too or much too mesially located.

Furthermore, for situations where it is more adequate to evaluate the two directional noncoverages, MNR and DNR, (e.g., for CIs constructed when extremal observation exist), values of DNR (MNR, resp.) between $\alpha \times 0.375$ and $\alpha \times 0.625$ correspond to CI methods which yield intervals with a satisfactory mesial (distal) location and values of DNR (MNR, resp.) outside that range will correspond to non-satisfactory mesially (distally) located intervals (for the nominal level $\alpha=0.05$, see Fig. 2 (b); more details in [13]). 


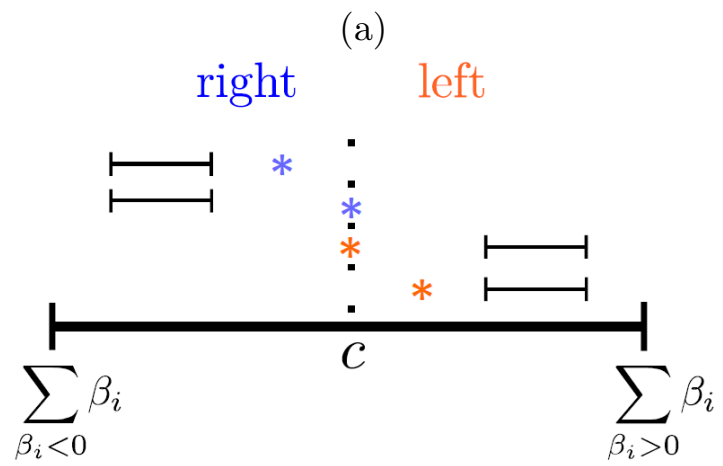

(b)

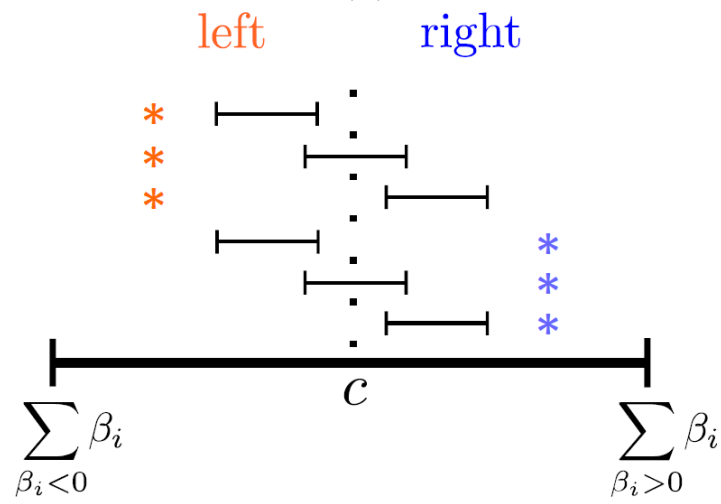

Figure 1: Examples of CIs located too distally (a) and too mesially (b) relatively to the true value $\mathrm{L}$ (identified by an asterisk), on the support scale of the linear combination. The midpoint of the support scale is denoted by $c$. 
(a)

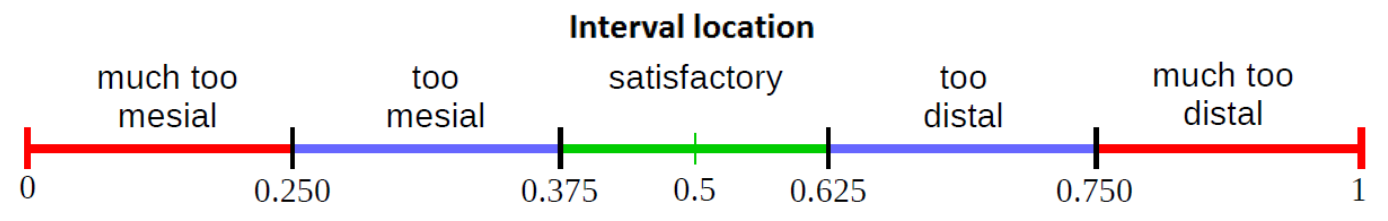

(b)

$\begin{array}{ccc}\text { Mesial location } \\ \text { non-satisfactory } & \text { satisfactory } & \text { Distal location } \\ \text { non-satisfactory } & \text { satisfactory } & \text { non-satisfactory } \\ 0.01875 & 0.025 & 0.03125\end{array}$

Figure 2: (a) Interpretation of interval location in terms of Q index. (b) Interpretation of the mesial and distal locations in terms of DNR and MNR. For a $95 \%$-confidence level, the CIs for a linear combination are expected to produce values of DRN and MNR between $(1-\mathrm{R}) \times 0.375=0.05 \times 0.375=0.01875$ and $(1-\mathrm{R}) \times 0.625=0.05 \times 0.625=0.03125$. 


\section{Graphical Representation}

In the evaluation of the performance of CI methods for a linear combination of $k \geq 2$ binomial proportions, $L=\sum_{i=1}^{k} \beta_{i} p_{i}$, over the whole or part of the parameter space, summary statistics of the exact or simulated coverage probabilities (e.g., [10, 13]) or twodimensional plots of the coverage probabilities, by holding one proportion $p_{i}$ and fixing the other ones (e.g., [4 for the difference of two proportions), are usually given. In addition to frequently not being able to draw the main conclusions in terms of the key parameter $L$, these types of summarizing procedures can also be misleading ([17).

In order to assess the performance of CI methods for a linear combination $L$, for any trial setting $n_{1}, n_{2}, \ldots, n_{k}$ and weights $\beta_{1}, \beta_{2}, \ldots, \beta_{k}$, we propose a different approach which consists of using a simple two-dimensional plot of DNR versus MNR. In this plot, all pairs $(\operatorname{MNR}(L), \operatorname{DNR}(L))$, computed via exact or Monte Carlo methods for different possible values of $L$, are represented by points. By inspecting the location of these points, we can visually check for the existence of desirable properties concerning R, MNR and DNR. Concretely, the following properties could be easily analyzed:

(i) The values of $\mathrm{R}$ should be close to the nominal level of $100(1-\alpha) \%$.

Since $1-\mathrm{R}=\mathrm{DNR}+\mathrm{MNR}$, then the closer the value of DNR + MNR is to $\alpha$ the better the performance of the $\mathrm{CI}$ in terms of coverage. Hence, the line DNR + $\mathrm{MNR}=\alpha$ and the two parallel lines, for instance DNR $+\mathrm{MNR}=\alpha+\varepsilon$ and DNR + $\mathrm{MNR}=\alpha-\varepsilon$, for some tolerance $\varepsilon>0$, allow us examining whether the coverage probabilities produced by the CI method under analyzed, for different values of the parameter $L$, are sufficiently close to the expected nominal level. Graphically, the points should be between the two border parallel lines;

(ii) When $\mathrm{R}$ values are below the nominal level, the CI method is classified as liberal. When $\mathrm{R}$ values are above the nominal level, the CI method is conservative.

Hence, graphically, having points above (below, respectively) the reference line DNR $+\mathrm{MNR}=\alpha$ means that the method produces more liberal (conservative, resp.) CIs;

(iii) The quantity MNR - DNR provides information about the balance between MNR and DNR. If MNR - DNR is close to 0 , the value of $\mathrm{Q}=\frac{\mathrm{MNR}}{\mathrm{MNR}+\mathrm{DNR}}$ will be around 0.5, which is interpreted as satisfactorily located CIs. Values of Q between the bounding references 0.375 and 0.625 will correspond to CI methods which yield intervals with a satisfactory location. Values of Q outside that range will correspond to non-satisfactory located intervals: less than 0.375 to intervals located too mesially to include the true value of $L$, and greater than 0.625 to intervals located too distally to include $L([13])$. Points between the two lines $\mathrm{DNR}=0.600 \mathrm{MNR}$ and $\mathrm{DNR}=$ 1.667 MNR will correspond to satisfactorily located CIs, since

$$
0.375 \leq \mathrm{Q} \leq 0.625 \Leftrightarrow 0.375 \leq \frac{\mathrm{MNR}}{\mathrm{MNR}+\mathrm{DNR}} \leq 0.625 \Leftrightarrow
$$




$$
\begin{gathered}
\frac{1-0.625}{0.625} \mathrm{MNR} \leq \mathrm{DNR} \leq \frac{1-0.375}{0.375} \mathrm{MNR} \Leftrightarrow \\
0.600 \mathrm{MNR} \leq \mathrm{DNR} \leq 1.667 \mathrm{MNR} .
\end{gathered}
$$

(iv) For situations in which extremal observations exist, it is convenient to apply CI methods for which it is expected that $\mathrm{MNR}=(1-\mathrm{R}) / 2=\alpha / 2$ and $\mathrm{DNR}=(1-$ $\mathrm{R}) / 2=\alpha / 2$. Values of DNR (MNR, resp.) between $\alpha \times 0.375$ and $\alpha \times 0.625$ will correspond to CI methods which yield intervals with a satisfactory mesial (distal, resp.) location. Values of DNR (MNR, resp.) outside that range will correspond to non-satisfactory mesially (distally, resp.) located intervals.

\section{$5 \quad$ Examples}

To illustrate the usefulness of the proposed graphical technique, we carried out a simulation study to show the advantages of using it to evaluate the coverage probabilities and interval location of the five variants of the adjusted Wald CIs for two types of linear combinations of $k=3$ independent binomial proportions: one with balanced weights, $\frac{1}{3} p_{1}+\frac{1}{3} p_{2}+\frac{1}{3} p_{3}$ (Scenario 1) and the other with unbalanced weights, $\frac{1}{3} p_{1}+\frac{1}{2} p_{2}+3 p_{3}$ (Scenario 2). Similar results were observed for others unbalanced and quasi-balanced configurations.

Using the Monte Carlo method, estimates of R, MNR, DNR and Q were obtained using 100 sets of 3 -samples simulated from binomial distributions, $\operatorname{Bin}\left(n_{i}, p_{i}\right), i=1,2,3$, where each $p_{i}$ is randomly generated from $U[0,1]$. Four configurations with different sample sizes $\left(n_{1}, n_{2}, n_{3}\right)$ were considered: $(10,10,10),(30,10,10),(30,20,10)$ and $(30,30,30)$. The quantities R, MNR, DNR and Q were computed for each parameter setting. Only the results for $95 \%$ will be herein discussed. All simulations were carried out using $\mathrm{R}$ software $(18])$.

For each Wald CI variant, average values of $\mathrm{R}, \mathrm{MNR}, \mathrm{DNR}$ and $\mathrm{Q}\left(\mathrm{R}_{\text {mean }}, \mathrm{MNR}_{\text {mean }}\right.$, $\mathrm{DNR}_{\text {mean }}$ and $\mathrm{Q}_{\text {mean }}$ ) were calculated for each parameter setting (Tables 1. 2). Based on these averaged quantities, we concluded the following:

$\mathbf{R}_{\text {mean }}$ : Wald CI variants-1, 2 yielded the best performances in terms of averaged coverage probabilities for both balanced and unbalanced weights;

Qmean: Wald variants-1,2 were also the best to produce CIs with satisfactory locations for most scenarios; Wald variant- 4 was found to be the worst in terms of absence of equilibrium between the mesial and distal non-coverage probabilities, particularly in unbalanced scenarios;

$\mathbf{M N R}_{\text {mean }}$ and $\mathbf{D N R}_{\text {mean }}$ : Wald variant-4 was the best to produce CIs with mesially satisfactory location and a very low probability of being distally located, for almost all scenarios. This result is consistent with the capability of this variant to handle extremal observations (i.e., when there are estimates of $p_{i}$ equal to 0 or 1 , for some i). 
Table 1: Results of averaged coverage and directional non-coverages for the five variants of the Wald CI, when $k=3$, for Scenarios 1 and 2. Confidence level $1-\alpha=95 \%$.

\begin{tabular}{|c|c|c|c|c|c|c|c|c|c|c|c|c|c|c|c|c|}
\hline \multirow[b]{3}{*}{$\left(\beta_{1}, \beta_{2}, \beta_{3}\right)$} & \multirow[b]{3}{*}{$n_{1} / n_{2} / n_{3}$} & \multicolumn{5}{|c|}{ variants } & \multicolumn{5}{|c|}{ variants } & \multicolumn{5}{|c|}{ variants } \\
\hline & & 0 & 1 & 2 & 3 & 4 & 0 & 1 & 2 & 3 & 4 & 0 & 1 & 2 & 3 & 4 \\
\hline & & \multicolumn{5}{|c|}{$\mathrm{R}_{\text {mean }}(\%)$} & \multicolumn{5}{|c|}{$\mathrm{MNR}_{\text {mean }}(\%)$} & \multicolumn{5}{|c|}{$\mathrm{DNR}_{\text {mean }}(\%)$} \\
\hline \multirow{4}{*}{$\left(\frac{1}{3}, \frac{1}{3}, \frac{1}{3}\right)$} & $10 / 10 / 10$ & 91.6 & 95.6 & 95.5 & 97.0 & 97.0 & 5.18 & 1.42 & 1.49 & 0.57 & 0.57 & 3.22 & 2.98 & 3.01 & 2.45 & 2.45 \\
\hline & $30 / 30 / 30$ & 94.0 & 95.2 & 95.2 & 95.6 & 95.6 & 3.47 & 1.95 & 1.98 & 1.62 & 1.62 & 2.53 & 2.85 & 2.82 & 2.74 & 2.74 \\
\hline & $30 / 10 / 10$ & 91.6 & 95.5 & 95.4 & 96.7 & 96.7 & 5.15 & 1.61 & 1.69 & 0.82 & 0.77 & 3.25 & 2.89 & 2.91 & 2.45 & 2.52 \\
\hline & $30 / 20 / 10$ & 92.2 & 95.4 & 95.3 & 96.4 & 96.3 & 4.75 & 1.74 & 1.82 & 1.06 & 1.03 & 3.05 & 2.86 & 2.88 & 2.53 & 2.65 \\
\hline \multirow{4}{*}{$\left(\frac{1}{3}, \frac{1}{2}, 3\right)$} & $10 / 10 / 10$ & 86.1 & 95.3 & 95.2 & 96.8 & 96.7 & 14.24 & 2.59 & 2.70 & 1.16 & 0.33 & 2.20 & 2.12 & 2.10 & 2.07 & 2.90 \\
\hline & $30 / 30 / 30$ & 91.1 & 95.0 & 94.9 & 95.5 & 96.0 & 7.16 & 3.04 & 3.14 & 2.53 & 1.18 & 1.74 & 1.97 & 1.96 & 1.92 & 2.85 \\
\hline & $30 / 10 / 10$ & 83.4 & 95.2 & 95.1 & 96.7 & 96.7 & 14.38 & 2.65 & 2.77 & 1.54 & 0.33 & 2.22 & 2.15 & 2.13 & 1.20 & 2.95 \\
\hline & $30 / 20 / 10$ & 82.4 & 95.1 & 95.0 & 96.6 & 96.7 & 15.45 & 2.74 & 2.86 & 1.25 & 0.34 & 2.15 & 2.16 & 2.14 & 2.12 & 3.00 \\
\hline
\end{tabular}


Table 2: Results of averaged index $\mathrm{Q}$ for the five variants of the Wald CI, when $k=3$, for Scenarios 1 and 2. Confidence level $1-\alpha=95 \%$.

\begin{tabular}{lcccccc}
\hline & & \multicolumn{5}{c}{ variants } \\
\cline { 3 - 7 }$\left(\beta_{1}, \beta_{2}, \beta_{3}\right)$ & $n_{1} / n_{2} / n_{3}$ & \multicolumn{5}{c}{3} \\
\hline \multirow{2}{*}{$\left(\frac{1}{3}, \frac{1}{3}, \frac{1}{3}\right)$} & $10 / 10 / 10$ & 0.617 & 0.322 & 0.332 & 0.188 & 0.188 \\
& $30 / 30 / 30$ & 0.579 & 0.406 & 0.413 & 0.371 & 0.371 \\
& $30 / 10 / 10$ & 0.613 & 0.358 & 0.367 & 0.250 & 0.234 \\
& $30 / 20 / 10$ & 0.609 & 0.379 & 0.387 & 0.296 & 0.281 \\
$\left(\frac{1}{3}, \frac{1}{2}, 3\right)$ & & & & & & \\
& $10 / 10 / 10$ & 0.792 & 0.550 & 0.562 & 0.359 & 0.101 \\
& $30 / 30 / 30$ & 0.805 & 0.607 & 0.615 & 0.568 & 0.293 \\
& $30 / 10 / 10$ & 0.866 & 0.553 & 0.566 & 0.364 & 0.102 \\
& $30 / 20 / 10$ & 0.878 & 0.559 & 0.572 & 0.372 & 0.102 \\
\hline
\end{tabular}

For each scenario and each variant of the Wald method, all the pairs of estimated values $(\mathrm{MNR}, \mathrm{DNR})$ and the centroid $\left(\mathrm{MNR}_{\text {mean }}, \mathrm{DNR}_{\text {mean }}\right)$ were displayed in a plot (see Fig. 3 for variants-0,4). The centroids correspond to values shown in Tables 1, 2. Based on these plots, we concluded:

R: All the classic Wald CIs (variant-0) constructed were very conservative (points above the line with negative slope). Some Wald variant-4 CIs exhibited good performance in terms of coverage (points close to the line with negative slope);

Q: Only Wald variant-0 tends to produce more satisfactorily located CIs when the weights are balanced (points between the two border lines with positive slopes);

MNR and DNR: Although the location of the centroids indicates that the averaged values of DNR have a satisfactory level for all situations represented in Fig. 3, there are some CIs produced by variant- 0 and variant- 4 of the adjusted Wald method that fall outside the region corresponding to a mesially satisfactory location. Therefore, one should be aware that the averaged values of DNR and MNR may not always be reliable indicators of interval location.

\section{Conclusions}

Using the shrinkage estimator $\left(X_{i}+h_{i}\right) /\left(n_{i}+2 h_{i}\right), h_{i}>0$, in the estimation of the proportion $p_{i}$, several types of adjustments of the Wald CI for a linear combination of $k$ independent binomial proportions can be constructed. To fully characterize the location of these different variants of the adjusted Wald CI and determine how satisfactory is their mesial (distal) location, particularly in situations in which extremal observations can exist, the mean of evaluation measures like the Q index, the MNR and the DNR may not be 
(a)

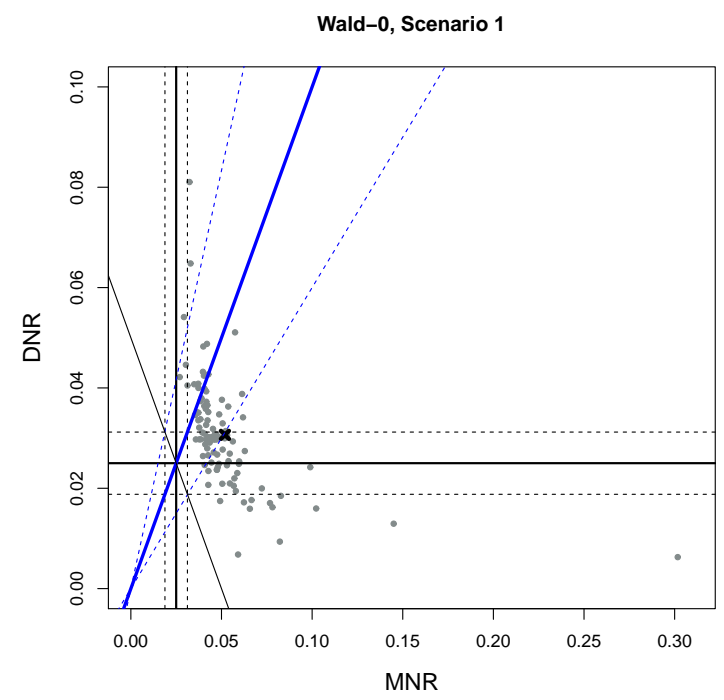

(c)

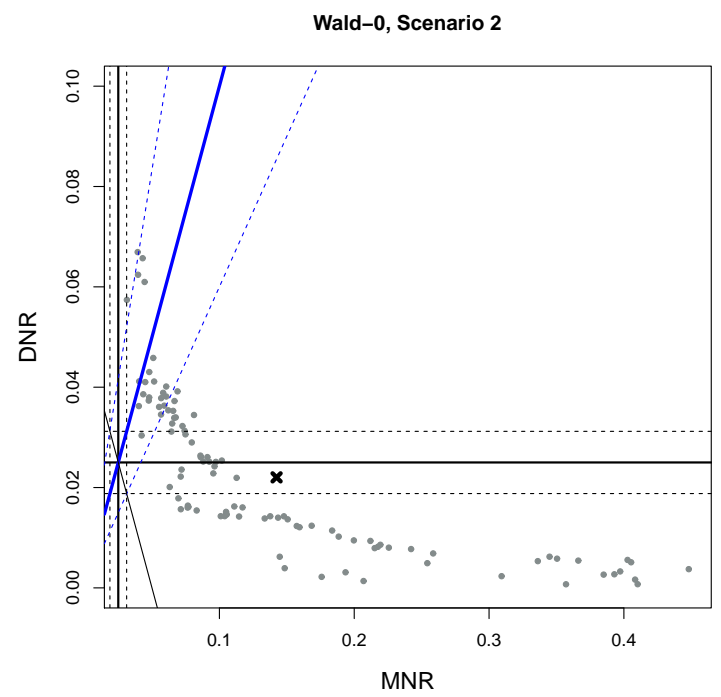

(b)

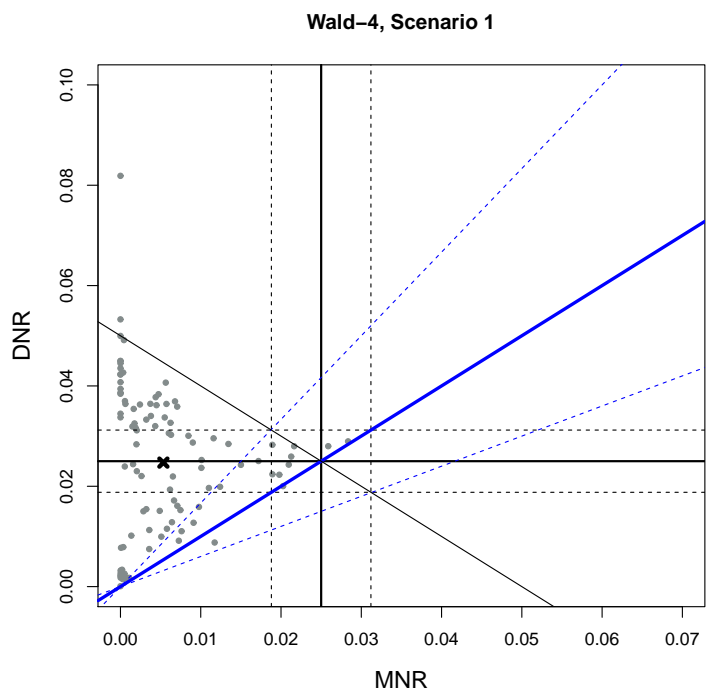

(d)

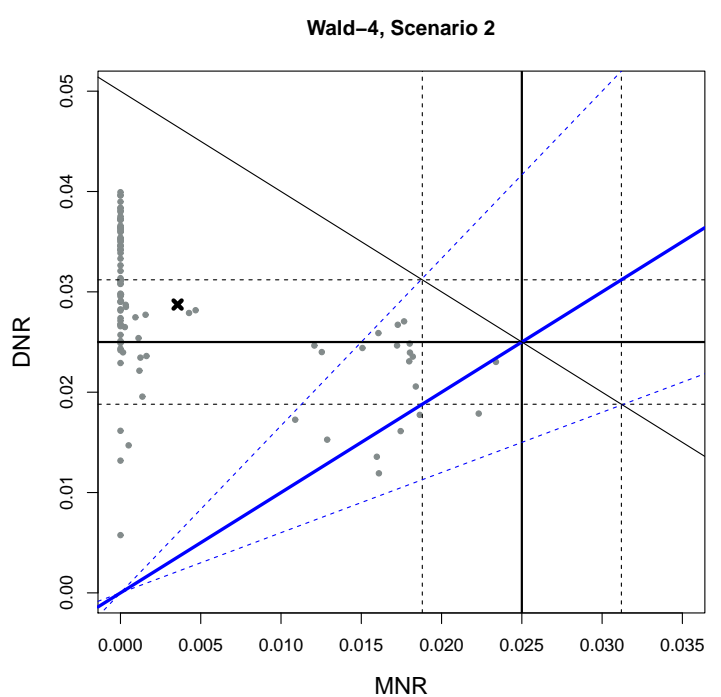

Figure 3: Plots of DNR vs MNR for variant-0 and variant-4 of the adjusted Wald CI, for the linear combinations $\frac{1}{3} p_{1}+\frac{1}{3} p_{2}+\frac{1}{3} p_{3}$ (Scenario 1, (a) and (b)) and $\frac{1}{3} p_{1}+\frac{1}{2} p_{2}+3 p_{3}$ (Scenario 2, (c) and (d)), in both cases for the sample sizes $(10,10,10)$. The centroid is marked by a cross. The solid lines are the reference lines associated to $\mathrm{R}$ (lines with negative slope), Q (lines with positive slope), MNR (vertical lines) and DNR (horizontal lines). The dashed lines bound the region around the corresponding reference lines where the interval location is classified as satisfactory in terms of Q, DNR and DNR. 
sufficient. In fact, these summarized statistics do not describe how well the values of $R$, MNR and DNR are distributed for different values of the key parameter $L$.

Our examples show that a graphical representation of the DNR vs MNR is easier to interpret, facilitating the evaluation of the location of the CIs. Moreover, this type of representation draws the attention to the need of analyzing the dispersion of the values of MNR and DNR in such evaluations.

\section{Acknowledgments.}

This work was partially supported by Portuguese funds through the CIDMA - Center for Research and Development in Mathematics and Applications, and the Portuguese Foundation for Science and Technology (FCT - Fundação para a Ciência e a Tecnologia), within project UID/MAT/04106/2013.

\section{References}

[1] Anbar, D.: On Estimating the Difference Between Two Proportions, with Special Reference to Clinical Trials. Biometrics 39, 257-262 (1983)

[2] Mee, R.: Confidence Bounds For the Difference Between Two Proportions. Biometrics 40, 1175-1176 (1984)

[3] Newcombe, R.: Interval Estimation for the Difference Between Independent Proportions: Comparison of Eleven Methods. Statistics in Medicine 17, 873-890 (1998)

[4] Agresti, A., Caffo, B.: Simple and Effective Confidence Intervals for Proportions and Differences of Proportions Result from Adding Two Successes and two Failures. The American Statistician 54, 280-288 (2000)

[5] Brown, L., Li, X.: Confidence intervals for two sample binomial distribution. Journal of Statistical Planning and Inference 130, 359-375 (2005)

[6] Fagerland, M., Lydersen, S., Laake, P.: Recommended Confidence Intervals for Two Independent Binomial Proportions. Statistical Methods in Medical Research 0, 1-31 (2011)

[7] Decrouez, G., Robison, A.: Confidence Intervals for the Weighted Sum of Two Independent Binomial Proportions. Aust. N. Z. Stat. 54, 281-299 (2012)

[8] Martín Andrés, A., Álvarez Hernández, M.: Optimal Method for Realizing TwoSided Inferences About a Linear Combination of Two Proportions. Communications in Statistics - Simulation and Computation 42, 327-343 (2013), DOI: 10.1080/03610918.2011.650263 
[9] Tebbs, J., Roths, S.: New large-sample confidence intervals for a linear combination of binomial proportions. Journal of Statistical Planning and Inference 138, 1884-1893 (2008)

[10] Price, R., Bonett, D.: An improved confidence interval for a linear function of binomial proportions. Computational Statistics \& Data Analysis 45, 449-456 (2004)

[11] Martín Andrés, A., Álvarez Hernández, M., Herranz Tejedor, I.: Inferences about a linear combination of proportions. Statistical Methods in Medical Research 20, 369-387 (2011), DOI: 10.1177/0962280209347953. Erratum in Statistical Methods in Medical Research 21(4), 427-428, (2012), DOI: 10.1177/0962280211423597

[12] Martín Andrés, A., Herranz Tejedor, I., Álvarez Hernández, M.: The optimal method to make inferences about a linear combination of proportions. Journal of Statistical Computation and Simulation 82, 123-135 (2012), DOI: 10.1080/00949655.2010.530601

[13] Escudeiro, S., Freitas, A., Afreixo, A.: Approximate confidence intervals for a linear combination of binomial proportions: A new variant. Communication in Statistics Simulation and Compution (2016), DOI: 10.1080/03610918.2016.1241408

[14] Agresti, A., Coull, B.: Approximate Is Better than Exact for Interval Estimation of Binomial Proportions. The American Statistician 52, 119-126 (1998)

[15] Newcombe, R.: Measures of Location for Confidence Intervals for Proportions. Communications in Statistics: Theory and Methods 40, 1743-1767 (2011)

[16] Newcombe, R.: Confidence Intervals for Proportions and Related Measures of Effect Size. CRC Press (2013)

[17] Laud, P., Daneb, A.: Confidence intervals for the difference between independent binomial proportions: comparison using a graphical approach and moving averages. Pharmaceut. Statist. 13, 294-308 (2014)

[18] R Core Team: R: A Language and Environment for Statistical Computing. R Foundation for Statistical Computing (2016) 